\title{
TANGLED EMBEDDINGS OF ONE-DIMENSIONAL CONTINUA
}

\author{
D. R. MCMILLAN, JR. ${ }^{1}$ AND HARRY ROW ${ }^{2}$
}

1. Introduction. Let $X$ be a 1-dimensional continuum (i.e. compact, connected metric space) in the 3 -sphere $S^{3}$, and let $A$ be a polygonal arc in $S^{3}$ with endpoints not in $X$. Intuitively, it seems reasonable to expect that there should exist an ambient homeomorphism which moves no point outside a small neighborhood of $X$, and which pushes $A$ into the complement of $X$. Supporting this belief are these facts: $X$ can be homeomorphically approximated as closely as desired by a continuum for which this conjecture is true (this follows from an examination of the proof on p. 529 of [4]); and the conjecture can be proven in many special cases, including the important one in which $X$ is locally contractible (see [2, Theorem 7] and [5, Lemma $3]$ ). We give here (Theorems 2 and 3 ) an embedding in $S^{3}$ of the Menger universal 1-dimensional curve which contradicts our intuition. ${ }^{3}$ Another interesting property is that our continuum cannot be homotopically linked by any unknotted simple closed curve in $S^{3}$. Our example also demonstrates that the cube-with-handles property (see below) is not topologically invariant.

The original motivation for studying the above question is given in [5, Lemma 2], where the equivalence between the "arc pushing" property and the cube-with-handles property is noted. ${ }^{4} \mathrm{~A}$ continuum $X$ in the interior of a piecewise-linear 3-manifold $M^{3}$ is said to have the cube-with-handles property if $X=\bigcap_{i=1}^{\infty} H_{i}$, where $H_{i}$ is a polyhedral cube-with-handles (i.e., 3-cell plus orientable handles of index one) in $M^{3}$ and $H_{i+1} \subset$ Int $H_{i}$. If each $H_{i}$ can be chosen to be a 3 -cell, then $X$ is cellular in $M^{3}$.

Presented to the Society, August 28, 1968; received by the editors July 1, 1968.

${ }^{1}$ Partially supported by grant NSF GP-8501.

2 Partially supported by grant NSF GP-7085.

${ }^{3}$ It has been pointed out to the authors that H. G. Bothe [Ein eindimensionales Kompaktum im $E^{3}$, das sich nicht lagetreu in die Mengersche Universalkurve einbetten lässt, Fund. Math. 54 (1964), 251-258] has constructed a similar embedding of the Menger universal one-dimensional curve. Bothe's techniques can be extended to show his embedding is tangled.

${ }^{4}$ Closely related is a "strong arc pushing" property where we require in addition that the ambient homeomorphism can be chosen arbitrarily close to the identity as in Lemma 3 of [5]. Bothe shows that his example fails to have this stronger property. It is not known to the authors whether these properties are equivalent for one-dimensional continua. 
The recognition of cellular sets is important in many topological problems. One convenient way to show that a set is cellular in $M^{3}$ is to prove first that it has the cube-with-handles property (see, e.g., [5, Corollary 1.3]), and then use the cellularity criterion (see the statement of Theorem 5) to "cut the handles." Although our example frustrates the first step in this approach for a 1-dimensional continuum, we find (Theorem 4 ) that a 1-dimensional continuum in an arbitrary $M^{3}$ does have small neighborhoods which are irreducible in the sense that each polyhedral 2-sphere in the neighborhood bounds a 3-cell in the neighborhood. This is sufficient for the effectiveness of the cellularity criterion.

Manifolds are always assumed to be connected, unless the contrary is explicitly stated.

2. Removing knotted tunnels. Let us say that a nonempty compact set $X$ in $S^{3}$ is tangled if each polyhedral, unknotted (in $S^{3}$ ) simple closed curve in $S^{3}-X$ is contractible to a point in $S^{3}-X$. If $X_{1}$, $X_{2}, \cdots$, is a sequence of tangled compact sets in $S^{3}$, and if $X_{i+1} \subset X_{i}$ for each $i \geqq 1$, then clearly $\bigcap_{i=1}^{\infty} X_{i}$ is tangled. A single point is an example of a tangled set, but we are more interested in the case where $S^{3}-X$ is not simply connected. Hence, a more significant example of a tangled set is a cube-with-a-knotted-hole (the $X$ in Lemma 1 below). If the simple closed curve of Lemma 1 were not known to be knotted, we might call $X$ a cube-with-a-hole.

LEMMA 1. Let $U$ be a regular neighborhood of a knotted simple closed curve in $S^{3}$. Then each polyhedral unknotted simple closed curve in Int $U$ bounds a polyhedral 2-cell in Int $U$. Hence, $S^{3}$ - Int $U=X$ is tangled.

Proofs of this and the next lemma are given in [6].

Lemma 2. Let $X$ be a tangled compact set in $S^{3}$ which has the cubewith-handles property. Then $S^{3}-X$ is a contractible set.

Let $L^{3}$ be a compact, polyhedral 3-manifold with nonempty boundary, and suppose $B^{3}$ is a polyhedral 3 -cell such that $B_{3} \cap L^{3}$ is an annulus $A$ in $\left(\operatorname{Bd} B^{3}\right) \cap\left(\operatorname{Bd} L^{3}\right)$. Let $K$ be a polyhedral spanning arc of $B^{3}$ joining the two components of $\left(\mathrm{Bd} B^{3}\right)-A$. We suppose further that $K$ is knotted relative to $B^{3}$. That is, there exists no polyhedral arc $K^{\prime}$ in $\mathrm{Bd} B^{3}$ joining the endpoints of $K$ such that $K \cup K^{\prime}$ bounds a polyhedral 2-cell in $B^{3}$. Let $T^{3}$ be a small regular neighborhood of $K$ in $B^{3}-A$, with $T^{3} \cap \mathrm{Bd} B^{3}$ a regular neighborhood in $\mathrm{Bd} B^{3}$ of Bd $K$. Letting $M^{3}$ denote $L^{3} \cup B^{3}$, we say that the 3-manifold 


$$
N^{3}=\mathrm{Cl}\left(M^{3}-T^{3}\right)
$$

is obtained from $M^{3}$ by removing a knotted tunnel. The tunnel is the 3-cell $T^{3}$. If $N^{3}$ is obtained from $M^{3}$ by removing a finite number of knotted tunnels whose associated $B^{3}$ 's are disjoint, we say that $N^{3}$ is obtained from $M^{3}$ by removing disjoint, unlinked knotted tunnels.

If we remove the requirement that $K$ be knotted relative to $B^{3}$, we may then speak of simply removing a tunnel from $M^{3}$ to obtain $N^{3}$ (i.e., if $M^{3}$ is $N^{3}$ plus a handle of index two). We may also say that $N^{3}$ is obtained from $L^{3}$ by attaching a cube-with-a-(knotted)-hole along $A$. This last statement will always be understood to imply that if we attach the cube-with-a-(knotted)-hole to a 3-cell along the same annulus $A$, then the result is a 3-cell (see Lemma 3 ).

Theorem 1. Suppose that $M^{3}$ and $N^{3}$ are compact, polyhedral 3-manifolds in $S^{3}$ such that $N^{3}$ is obtained from $M^{3}$ by removing a knotted tunnel. Then $M^{3}$ is tangled if and only if $N^{3}$ is tangled.

Proof. We use the notation introduced in defining "removing a knotted tunnel." Assume first that $M^{3}$ is tangled. Let $\Delta$ denote a fixed component of $T^{3} \cap \mathrm{Bd} B^{3}$. Then $\Delta$ is a 2-cell in $\mathrm{Bd} M^{3}$, $\Delta \cap N^{3}=\operatorname{Bd} \Delta$, and $N^{3} \cup \Delta$ is a tangled set.

Now suppose that $J$ is a polyhedral, unknotted simple closed curve in $S^{3}-N^{3}$. After performing a small ambient isotopy of $J$, we may assume that $J$ and $\Delta$ are in general position. Proof is by induction on the number of points, $n(J)$, in $J \cap \Delta$. By Lemma $1, J=\operatorname{Bd} D$ where $D$ is a polyhedral 2-cell missing the cube-with-a-knotted-hole $\mathrm{Cl}\left(B^{3}-T^{3}\right)$. In particular, $D \cap \mathrm{Bd} \Delta=\varnothing$. We suppose that $D$ and $\Delta$ are in general position, so that each of the finite number of components of $D \cap \Delta$ is either a polygonal simple closed curve in Int $D$ or a polygonal spanning arc of $D$. Note that $n(J)$ is twice the number of components of $D \cap \Delta$ which are arcs.

If $n(J)=0$, then $J \cap\left(N^{3} \cup \Delta\right)=\varnothing$ and so $J$ is contractible in $S^{3}-\left(N^{3} \cup \Delta\right)$. If $n(J)>0$, then there is an arc $Z \subset D \cap \Delta$ which divides $D$ into polyhedral 2-cells $D_{1}, D_{2}$ such that $D_{1} \cap D_{2}=Z$ and $\Delta \cap B d$ $D_{1}=Z$. Hence $J$ is homotopic in $S^{3}-N^{3}$ to an unknotted simple closed curve $J^{*}$ with $n\left(J^{*}\right)=n(J)-2$. Specifically, take $J^{*}$ to be the boundary of: $D$ minus a small neighborhood in $D$ of $D_{1}$. This completes the proof in one direction.

The converse follows immediately from the fact that $N^{3} \subset M^{3}$ and that the inclusion of $S^{3}-M^{3}$ into $S^{3}-N^{3}$ induces a monomorphism on fundamental groups. Details are left to the reader. 
3. A tangled one-dimensional Menger universal curve. We will construct our continuum from a 3 -simplex in $S^{3}$ by removing knotted tunnels and the interiors of polyhedral 3-cells. To simplify notation we will denote a complex and its geometric carrier by the same symbol. Let $K_{0}$ be the complex consisting of a 3-simplex and its faces. For $n \geqq 1$, let $K_{n-1}^{(2)}$ be the standard second barycentric subdivision of $K_{n-1}$.

Let the $B_{n-1}^{i}$ 's be the disjoint 3-cells we obtain by taking stars of barycenters of 3-simplexes of $K_{n-1}$ in $K_{n-1}^{(2)}$. Call $Q_{n}$ the 3-manifold

$$
\mathrm{Cl}\left(K_{n-1}^{(2)}-\cup B_{n-1}^{i}\right) \text {. }
$$

$Q_{n}$ is the standard second derived regular neighborhood of the 2 -skeleton of $K_{n-1}$. Let the $C_{n-1}^{t}$ 's be the disjoint spanning 3-cells of $Q_{n}$ we obtain by taking stars of barycenters of 2 -simplexes of $K_{n-1}$ in $Q_{n}$. Let $P_{n}$ be the standard second derived regular neighborhood of the 1-skeleton of $K_{n-1}$. Each $C_{n-1}^{i}$ meets $P_{n}$ in an annulus $A_{n-1}^{i}$. Let $T_{n-1}^{i}$ be a knotted tunnel in $Q_{n}$ associated with $C_{n-1}^{i}$ and $A_{n-1}^{i}$. Then

$$
\mathrm{Cl}\left(Q_{n}-U T_{n-1}^{i}\right)
$$

is a 3-manifold $K_{n}$. More precisely, choose for $K_{n}$ a subcomplex of a subdivision of $Q_{n}$. We can think of $K_{n}$ as being obtained from $P_{n}$ by attaching cubes-with-knotted-holes along the $A_{n-1}^{i}$ 's. Note that the mesh of the complex $K_{n}$ approaches zero as $n$ increases. Let $K=$ $\bigcap_{n=1}^{\infty} K_{n}$.

Theorem 2. $K$ is tangled and $S^{3}-K$ is not simply connected. Hence $K$ fails to have the cube-with-handles-property.

Proof. $K_{n}$ is obtained from $K_{n-1}$ by removing the interiors of disjoint 3 -cells and then removing disjoint and unlinked knotted tunnels. $Q_{1}$ is tangled. Using Theorem 1 , clearly $K_{1}$ is tangled. Continuing in this manner, $K_{n}$ is tangled for each $n$. Hence $K=\bigcap_{n=1}^{\infty} K_{n}$ is tangled.

$K_{1}$ is a 3-simplex with the interior of a polyhedral 3-cell and four disjoint and unlinked knotted tunnels removed. There is a simple closed curve in $S^{3}-K_{1}$ that is not contractible in the complement of the 1 -skeleton of $K_{0}$. Since $K$ contains the 1 -skeleton of $K_{0}, S^{3}-K$ is not simply connected.

Lemma 2 shows that $K$ does not have the cube-with-handles property. 
Theorem 3. $K$ is a one-dimensional Menger universal curve.

Proof. We will show $K$ is 1 -dimensional by $\epsilon$-mapping $K$ into a finite graph. Let $\epsilon>0$. Choose $K_{n-1}$ so that mesh $K_{n-1}<\epsilon / 8$. Now $K_{n}$ is obtained from $P_{n}$ by attaching a finite number of cubes-withknotted-holes $\mathrm{Cl}\left(C_{n-1}^{i}-T_{n-1}^{i}\right)$ along $A_{n-1}^{i}$. Using a technique of Hempel in [3, Theorem 5], we can retract $\mathrm{Cl}\left(C_{n-1}^{i}-T_{n-1}^{i}\right)$ onto $A_{n-1}^{i}$. Hence there is a retraction $r_{1}$ from $K_{n}$ onto $P_{n}$ that moves no point more than the maximum diameter of the $C_{n-1}^{i}$ 's, i.e., less than $\epsilon / 4$.

$P_{n}$ is the standard second derived regular neighborhood of the 1 -skeleton, $R$, of $K_{n-1}$. So there is a retraction $r_{2}$ of $P_{n}$ onto $R$ that moves points only within simplices of $K_{n-1}$. Hence $r_{2}$ moves points less than $\epsilon / 8$. Thus $r_{2} r_{1}$ is a retraction of $K_{n}$ onto $R$ that moves points less than $\epsilon / 2$. So $r_{2} r_{1}$ is an $\epsilon$-map. Hence $r_{2} r_{1} \mid K$ is the required map.

R. D. Anderson [1, Theorem XII] has shown that any 1-dimensional Peano continuum with (1) no local cut points and (2) the property that each open subset contains the 1-skeleton of a 4-simplex, is homeomorphic to Menger's 1-dimensional universal curve. We leave to the reader the task of checking these conditions.

4. Irreducible neighborhoods of one-dimensional continua. The previous example shows that one cannot expect to find small cubewith-handles neighborhoods of an arbitrary 1-dimensional continuum in $S^{3}$. Theorem 4 of this section shows how to construct neighborhoods which are irreducible. We then give in the corollary to Theorem 5 an instance in which irreducible neighborhoods work as well as cubes-with-handles.

Lemma 3. Suppose $M^{3}$ and $N^{3}$ are compact polyhedral 3-manifolds such that $N^{3}$ is obtained from $M^{3}$ by attaching a cube-with-a-hole $C$ along an annulus $A$ that is contractible in $M^{3}$. Then $M^{3}$ is homeomorphic to $N^{3}$.

Proof. Let $J$ be a polyhedral simple closed curve in Int $A$ that is not contractible in $A$. Now $J$ is contained in Bd $M^{3}$ so by Dehn's Lemma there is a polyhedral spanning 2-cell $D$ in $M^{3}$ with $J$ as boundary. Let $U^{3}$ be a regular neighborhood of $D$ in $M^{3}$ such that $U^{3} \cap \mathrm{Bd} M^{3}=A$. Let

$$
Q=\mathrm{Cl}\left(M^{3}-U^{3}\right)=\mathrm{Cl}\left(N^{3}-\left(U^{3} \cup C\right)\right) .
$$

Note that $U^{3} \cap Q$ and $\left(U^{3} \cup C\right) \cap Q$ both consist of the same disjoint pair of 2-cells and that both $U^{3}$ and $U^{3} \cup C$ are 3 -cells. In fact, there 
is a homeomorphism of $U^{3}$ onto $U^{3} \cup C$ which is the identity on $U^{3} \cap Q$. Hence, $M^{3}$ and $N^{3}$ are homeomorphic.

Leмma 4. Let $M^{3}$ and $N^{3}$ be compact polyhedral 3-manifolds and suppose $N^{3}$ is obtained from $M^{3}$ by attaching a cube-with-a-hole $C$ along an annulus $A$. Then $N^{3}$ is irreducible if and only if $M^{3}$ is irreducible.

Proof. Suppose $N^{3}$ is irreducible. Let $S$ be a polyhedral 2-sphere in Int $M^{3}$. Then $S$ bounds a 3 -cell in $N^{3}$ which cannot meet $C$. So $S$ bounds a 3 -cell in $M^{3}$.

Now suppose $M^{3}$ is irreducible. If $A$ were contractible in $M^{3}$ then by Lemma $3, M^{3}$ is homeomorphic to $N^{3}$. Suppose $A$ is not contractible in $M^{3}$. Note also that $A$ is not contractible in $C$. Let $S$ be a polyhedral 2-sphere in Int $N^{3}$. By a small isotopy put $A$ and $S$ in general position so that $A \cap S$ consists of a finite number of disjoint polyhedral simple closed curves $J_{1}, \cdots, J_{k}$. Now $C$ and $M^{3}$ are both irreducible so if $A \cap S=\varnothing$, then $S$ bounds a 3-cell in $N^{3}$. If $A \cap S \neq \varnothing$, select $J_{i}$ so that $J_{i}$ bounds a 2 -cell $D$ on $S$ missing the other $J_{j}$ 's. But $J_{i}$ bounds a 2 -cell $D^{\prime}$ in $A$ since $A$ is contractible in neither $C$ nor $M^{3}$. Hence $D \cup D^{\prime}$ is a 2 -sphere in $C$ or in $M^{3}$, so it bounds a 3 -cell $B^{3}$. We can remove the intersection curve $J_{i}$ (and possibly others) by isotoping $D$ slightly past $D^{\prime}$ using $B^{3}$ as a guide. By induction on the number of simple closed curves in $A \cap S$, we see that $S$ bounds a 3 -cell in $N^{3}$.

THEOREM 4. If $X$ is a one-dimensional compact set in the interior of a compact polyhedral 3-manifold with nonempty boundary $M^{3}$, then by removing a finite number of disjoint, unlinked knotted tunnels we obtain an irreducible 3-manifold $N^{3}$ whose interior contains $X$.

Proof. Notice that we do not assume $M^{3}$ orientable. If $M^{3}$ is nonorientable, we interpret "cube-with-handles" below as "regular neighborhood of a finite, connected graph." $M^{3}$ has a Heegaard decomposition consisting of a cube-with-handles $H$ and a finite number of disjoint 3-cells $C_{1}, \cdots, C_{k}$ such that

$$
M^{3}=H \cup\left(\bigcup_{i=1}^{k} C_{i}\right)
$$

and $H \cap C_{i}=(\operatorname{Bd} H) \cap\left(\operatorname{Bd} C_{i}\right)=A_{i}$, an annulus.

Now $X \cap C_{i}$ does not separate the two components of $\mathrm{Bd} C_{i}-A_{i}$ in $C_{i}$. Hence, we can find a polyhedral spanning arc $K_{i}$ of $C_{i}$ that joins the two components of $\mathrm{Bd} C_{i}-A_{i}$ in $C_{i}$ and misses $X$. We leave 
to the reader the easy task of insuring that $K_{i}$ is knotted relative to $C_{i}$. Let $T_{i}^{3}$ be a small regular neighborhood of $K_{i}$ in $C_{i}-A_{i}$ that misses $X$. Then $\mathrm{Cl}\left(M^{3}-\bigcup_{i=1}^{k} T_{i}^{3}\right)$ is the required 3 -manifold $N^{3}$. We can think of $N^{3}$ as being obtained from $H$ by attaching a finite number of disjoint cubes-with-knotted-holes. Since $H$ is irreducible, repeated application of Lemmas 3 and 4 shows that $N^{3}$ is irreducible.

REMARK. The $N^{3}$ constructed above can be shown to have other useful properties. For example, there is a mapping $f$ of $N^{3}$ onto a cube-with-handles $H$ such that $f \mid \mathrm{Bd} N^{3}$ is a homeomorphism of $\mathrm{Bd} N^{3}$ onto $\mathrm{Bd} H$.

Question. Does a 1-dimensional continuum in the interior of an orientable 3-manifold have small neighborhoods embeddable in $S^{3}$ ?

If $X$ is a continuum in the interior of a piecewise-linear $n$-manifold $M^{n}, n \geqq 3$, we say that $X$ satisfies the cellularity criterion in $M^{n}$ if: for each open set $U$ of $M^{n}$ containing $X$, there is an open set $V$ of $M^{n}$ such that $X \subset V \subset U$ and each loop in $V-X$ is contractible in $U-X$. A manifold is closed if it is compact and has no boundary.

THEOREM 5. Let $M^{3}$ be a piecewise-linear 3-manifold which is irreducible but not closed, and let $X \subset$ Int $M^{3}$ be a continuum which separates no connected open subset of $M^{3}$ which contains $X$. Then $X$ is cellular in $M^{3}$ if and only if $X$ satisfies the cellularity criterion.

Proof. Let $W$ be a given open set in Int $M^{3}$ containing $X$. Since $X$ separates no connected open subset of $M^{3}$, there are compact 3-manifolds $N^{3}$ and $R^{3}$, each with connected boundary, such that

$$
X \subset \text { Int } R^{3} \subset R^{3} \subset \text { Int } N^{3} \subset N^{3} \subset W,
$$

such that each loop in $R^{3}-X$ is contractible in $N^{3}-X$, and such that each loop in $N^{3}-X$ is contractible in $W-X$.

Since $M^{3}$ is not closed, each of $N^{3}$ and $R^{3}$ is irreducible, and $\mathrm{Bd} R^{3}$ represents a nontrivial element of $H_{2}\left(N^{3}-X ; Z\right)$. Further, it follows easily, from the fact that each loop in the orientable closed 2-manifold $\mathrm{Bd} R^{3}$ is contractible in $N^{3}-X$, that this cycle is spherical, i.e. can be represented by a singular 2 -sphere in $N^{3}-X$. Hence by [7, Theorem 1.1] there is an essential polyhedral 2 -sphere $\Sigma$ in the orientable 3-manifold $N^{3}-X$. Now $\Sigma$ bounds a 3 -cell in $N^{3} \subset W$, and its interior clearly must contain $X$. This completes the proof.

Corollary. Let $X$ be a one-dimensional continuum in the interior of a piecewise-linear 3-manifold $M^{3}$. Then $X$ is cellular in $M^{3}$ if and only if $X$ satisfies the cellularity criterion.

Proof. This is immediate from Theorems 4 and 5. 


\section{REFERENCES}

1. R. D. Anderson, One-dimensional continuous curves and a homogeneity theorem, Ann. of Math. 68 (1958), 1-16.

2. Steve Armentrout, Decompositions of $E^{8}$ with a compact 0-dimensional set of nondegenerate elements, Trans. Amer. Math. Soc. 123 (1966), 165-177.

3. John Hempel, $A$ surface in $S^{3}$ is tame if it can be deformed into each complementary domain, Trans. Amer. Math. Soc. 111 (1964), 273-287.

4. S. Lefschetz, On compact spaces, Ann. of Math. 32 (1931), 521-538.

5. D. R. McMillan, Jr., A criterion for cellularity in a manifold. II, Trans. Amer. Math. Soc. 126 (1967), 217-224.

6. Stephen Slack, Cellularity in certain 3-manifolds, $\mathrm{Ph} . \mathrm{D}$. Thesis, University of Wisconsin, Madison, 1968.

7. J. H. C. Whitehead, On 2-spheres in 3-manifolds, Bull. Amer. Math. Soc. 64 (1958), 161-166.

UNIVERSITY OF WISCONSIN 